\title{
Norois
}

Environnement, aménagement, société

Le restaurant, outil de développement local ?

\section{Verdier (Philippe), 2009. - Apprendre à faire la ville avec ses habitants}

\section{Anne-Sophie Charbonnier}

\section{(2) OpenEdition}

\section{Journals}

\section{Édition électronique}

URL : http://journals.openedition.org/norois/3615

DOI : $10.4000 /$ norois.3615

ISSN : $1760-8546$

\section{Éditeur}

Presses universitaires de Rennes

\section{Édition imprimée}

Date de publication : 30 juin 2011

Pagination : 131-132

ISBN : 978-2-7535-1479-9

ISSN : 0029-182X

\section{Référence électronique}

Anne-Sophie Charbonnier, « Verdier (Philippe), 2009. - Apprendre à faire la ville avec ses habitants », Norois [En ligne], 219 | 2011, mis en ligne le 30 septembre 2013, consulté le 22 septembre 2020. URL : http://journals.openedition.org/norois/3615 ; DOI : https://doi.org/10.4000/norois.3615

Ce document a été généré automatiquement le 22 septembre 2020.

(c) Tous droits réservés 


\title{
Verdier (Philippe), 2009. - Apprendre à faire la ville avec ses habitants
}

\author{
Anne-Sophie Charbonnier
}

\section{RÉFÉRENCE}

VERDIER (Philippe), 2009. - Apprendre à faire la ville avec ses habitants, Adels/Yves Michel, 264 p. ISBN 978-2-913492-69-1, 24,50€

1 Les frontières entre les différentes sciences tendraient-elles, avec le temps, à s'estomper? Ce livre est, en effet, une forme de cristallisation du partage des savoirs, s'inscrivant alors dans une démarche pluridisciplinaire. C'est un mélange subtil combinant dans un même temps, l'histoire de l'architecture et la sociologie urbaine, à travers un objet d'étude commun : celui de la ville. Les questionnements soulevés par Philippe Verdier à propos du projet urbain impliquent aujourd'hui tout type d'acteurs. De fait, la volonté de rendre plus accessible et plus compréhensif le devenir de nos villes, nous concerne tous. L'auteur, à la fois architecte et sociologue, réalise cet ouvrage dans un but précis, celui de « rendre lisible à des non-spécialistes des notions de base sur l'espace de la ville et de la demande sociale ». Cette réflexion sur la ville comporte deux dimensions essentielles: l'une spatiale, l'autre sociale. Ce qui est recherché par cette approche sociologique est précisément la mise en évidence des relations existantes entre les individus et l'organisation de leur espace. Celui-ci peut être corporel, l'espace est ainsi lié au corps, mais il peut également être simplement organisationnel, voué dès lors à la gestion de l'espace (public, privé, etc.). C'est dans cette double acceptation du terme que l'espace est, en tant que tel, objet de débat scientifique. Dans la première partie de l'ouvrage, plusieurs chronologies thématiques sont exposées de manière divertissante, à la fois sur l'espace mais aussi sur le moyen de l'appréhender en tant que dimension sociale à travers les différences culturelles. On retrouve ainsi les prémisses des courants socio-urbanistiques et architecturaux (à 
travers l'histoire, la société et la politique) concernant les villes et leurs constructions. Partant des chasseurs-cueilleurs ou des Pharaons à Thèbes, on arrive aux sociétés contemporaines qui pensent la ville de manière hétérogène. Différents courants servent en effet à penser la ville en tant qu'objet d'étude, on peut citer le courant fonctionnaliste ou encore le courant culturaliste. Par là, la ville est à la fois l'instrument du pouvoir en place et le reflet d'une multitude de données imperceptibles qu'il nous faut étudier. Dans la seconde partie de l'ouvrage, ce sont les diverses étapes au cœur de la constitution et de la formation d'un projet urbain qui sont présentées. Afin de faciliter la compréhension de ses lecteurs, l'auteur a choisi d'énumérer grâce à des tirets, les aspects plus ou moins positifs, et notamment ceux que les professionnels de la ville se doivent d'éviter. Toujours de manière assez succincte est évoquée l'importance de la prise en considération de la notion même de " projet urbain ». Cette notion, selon les disciplines et les professions, peut recouvrir plusieurs sens, ne permettant plus de rendre le travail pluridisciplinaire fructueux. Finalement, chacun des chapitres de ce livre comprend en son sein des illustrations, des dessins, des croquis mais aussi des tableaux rendant le peu de lecture agréable, animée et accessible à tous. "Apprendre à faire la ville avec ses habitants" sous-entend qu'une implication croissante des habitants dans la réalisation de projets urbains est possible. Cependant, l'auteur omet de consacrer un moment sur la faisabilité même d'inclure lesdits habitants dans la réalisation d'un projet urbain participatif. Bien qu'il puisse y avoir un effet pervers à vouloir faire participer le plus grand nombre d'habitants, puisqu'en partant de décisions individuelles, il serait difficile de répondre aux attentes de chacun dans un unique projet commun, nous sommes ici en droit d'attendre une recette à la croisée des disciplines dans l'objectif de faire participer au mieux chacun des habitants. Cette participation permettrait une meilleure réponse quant à leur propre confort, mais permettrait aussi d'établir une parité entre les aménageurs et les habitants. Il ne faudrait plus créer de frontière entre ceux qui décident des projets urbains, et ceux qui les subissent (prenons pour exemple les grands ensembles des années 1970) mais plutôt penser l'urbain comme une circularité d'idées, de réflexions et d'actions, entre les aménageurs et les habitants pour construire la ville sans la subir. Néanmoins, toute la difficulté résulte dans le fait de pouvoir faire combiner différents acteurs qui tentent d'élaborer un projet urbain sans avoir, nécessairement, les mêmes attentes. Bien souvent, les professionnels de la ville réalisent un projet urbain compris sur du long terme, a contrario des habitants qui sont, a priori, dans une perspective d'immédiateté. Par ailleurs, l'ouvrage ne nous permet pas de se rendre compte du poids réel des habitants dans la réalisation des projets urbains, que ce soit en termes d'influences sociales ou de résultats spatiaux. La position des habitants est parfois peu écoutée et c'est en ce sens que la participation des professionnels de la ville pourrait permettre une meilleure connaissance des fonctionnements « administratifs » à propos du projet urbain. D'un point de vue sociologique, l'accent aurait pu être davantage mis sur la difficulté qu'il y a de mettre en scène différents acteurs qui sous tendent à une hétérogénéité d'attentes.

2 Tout l'enjeu aurait été de sortir du schéma de pensée selon lequel nous pensons faire la ville de manière démocratique. Il semblerait qu'une réflexion concernant les accomodements de chacun des acteurs qui composent une ville, paraisse inévitable. De même, il faudrait comprendre comment la mise en relation ou l'absence de cette mise en relation, des professionnels de la ville, permettent, ou non, de trouver une satisfaction d'intérêts économique, social, culturel, spatial ou encore étatique. 


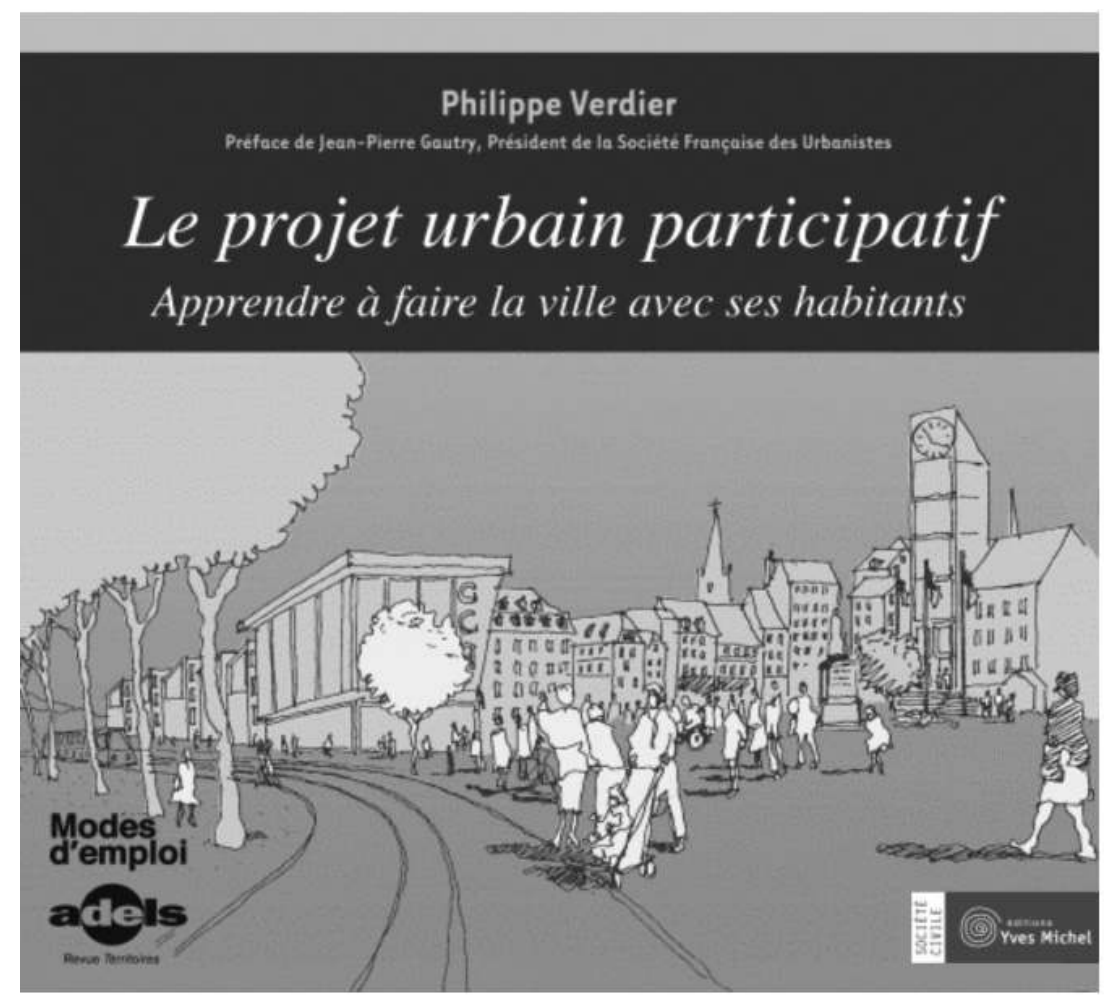

